\title{
Diagnóstico y tratamiento de la angina de Ludwig: reporte de un caso
}

\section{Diagnosis and treatment of Ludwig's Angina: a case report}

\section{Resumen}

La angina de Ludwig es una celulitis potencialmente mortal, rápidamente progresiva que involucra el piso de boca y la región cervico-facial. Se caracteriza por una induración y afectación del espacio submandibular, sublingual y submental. Cursa con alteraciones sistémicas y metabólicas. Por lo general, no se observan abscesos o linfadenopatías en la descripción clásica, sin embargo la progresión a la formación de abscesos dentro del espacio involucrado y los espacios contiguos es frecuente. La etiología en la mayoría de casos es odontogénica. El tratamiento debe priorizar el manejo de la vía aérea, drenaje quirúrgico, eliminación de la causa de infección, antibioticoterapia rápida agresiva y el control metabólico con soporte hídrico electrolítico. Se presenta el caso de una paciente mujer con antecedentes de anemia y odontalgia de pieza dentaria del maxilar inferior. Acudió por el servicio de emergencia con un aumento de volumen de tercio inferior facial, con predominio de región submandibular bilateral, submental y sublingual. Mal estado general, fiebre, taquicardia, disfagia, odinofagia y leucocitosis. Se diagnosticó angina de Ludwig. Se realizó el manejo de la vía aérea, drenaje intraoral, drenaje extraoral, exodoncia, antibioticoterapia inmediata y soporte hídrico electrolítico. Evolucionó favorablemente y posteriormente fue dada de alta sin complicaciones.

Palabras clave: Angina de Ludwig; Celulitis; Mandíbula.

\section{Caso Clínico}

Christian Farfán Gutiérrez ',a ${ }^{\text {, Marco Arce Lazo }}$ 2,a

1 Universidad Nacional Mayor de San Marcos.

Lima, Perú.

2 Hospital Regional Honorio Delgado. Arequipa,

Perú.

a Cirujano Bucal y Maxilofacial

\section{Correspondencia:}

Christian Farfán Gutiérrez

Correo electrónico: christianjr_88_@hotmail. com

Jirón Tarma 119 - Dpto. 502 - Cercado de Lima, Perú.

\section{Coautor:}

Marco Arce Lazo

asdent 7@hotmail.com

Conflicto de intereses: Los autores declaran no tener conflictos de interés.

Fuente de financiamiento: Autofinanciado

Fecha de recepción: 26/03/18

Fecha de aceptación: 24/04/18

\begin{abstract}
Ludwig's angina is a life-threatening, rapidly progressive cellulitis that involves the floor of the mouth and the cervico-facial region. With induration and involvement of bilateral submandibular space, sublingual bilateral and submental. It presents with systemic and metabolic alterations. In general, no abscess or lymphadenopathy is seen in the classic descriptionHowever, progression to abscess formation within the space involved and contiguous spaces is common. The etiology in most cases is odontogenic. Treatment should prioritize the management of the airway, surgical drainage, elimination of the cause of infection, aggressive rapid antibiotic therapy and metabolic control with electrolytic water support. A female patient with a history of anemia presented odontalgia of the lower jaw teeth. She came the emergency room with an increase in volume of the lower third of the face. Predominantly on submandibular bilateral, submental and sublingual regions, as well as scanted general condition, fever, tachycardia, dysphagia, odynophagia and leukocytosis. The diagnosis of Ludwig's angina was reached. Management of the airway, intraoral drainage, extraoral drainage, exodontia, immediate antibiotic therapy and electrolytic water support; were performed. She evolved favorably and was later discharged without complications.
\end{abstract}

Keywords: Cellulitis; Ludwig's angina; Mandible. 


\section{Introducción}

La angina de Ludwig es una celulitis bilateral rápidamente progresiva que involucra el espacio submandibular bilateral, sublingual bilateral y submental ${ }^{1}$. En la mayoría de casos se produce una protrusión lingual o desplazamiento posterior debido al compromiso del piso de la boca ${ }^{1}$.

Lleva el nombre del médico alemán Karl Friedrich Wilhelm Ludwig, debido a que reportó por primera vez esta condición en $1836^{2}$. Su descripción se basó en la observación de cinco pacientes con afectación de los tejidos conectivos del cuello, que rápidamente involucraron a los tejidos que cubren los músculos laríngeos y de la región sublingual. Las personas afectadas suelen tener entre 20 y 60 años, con predominio masculino, en una relación de $2: 1^{3}$.

Antes del desarrollo de la penicilina por Alexander Fleming y su producción en la década de 1950, la mortalidad asociada con la angina de Ludwig era preocupante $(50 \%)^{4}$. Actualmente, al existir alternativas quirúrgicas y formas de antibioticoterapia basadas en evidencia científica, la tasa de mortalidad por esta patología ha disminuido de un $50 \%$ a un $5 \%$. La etiología se atribuye en un $80 \%$ a pacientes con infecciones odontogénicas preexistentes ${ }^{6}$. Existen casos reportados posteriores a heridas a nivel sublingual, trauma facial, presencia de cuerpos extraños y sialodenitis de glándula submandibular $^{7}$. Los aislados bacterianos de la angina de Ludwig son variados, con predominio de aerobios y anaerobios, incluidos estreptococos $a$-hemolíticos, estafilococos y bacteroides ${ }^{8}$.

La mayoría de casos se presenta en pacientes con enfermedad sistémica existente ${ }^{1}$. Pacientes con diabetes mellitus, insuficiencia renal, cirrosis hepática, virus de la inmunodeficiencia humana, anemia y hábitos nocivos como el consumo de alcohol tienen un mayor riesgo de desarrollarla ${ }^{1}$. El diagnóstico se realiza sobre la base de la presentación clínica ${ }^{1}$. Signos y síntomas como disfagia, disnea, trismus y odontalgia son representativos de esta afección. La tomografía computarizada es útil para definir el alcance y la ubicación de la infección con predominio del mediastino 9. Toda la evidencia, independiente de los exámenes auxiliares, refuerza la importancia para valorar el compromiso de la vía aérea a través de un buen juicio clínico ${ }^{10}$.

Sin tratamiento inmediato, la infección puede extenderse rápidamente a los espacios mediastínicos causar una obstrucción de la vía aérea, fascitis necrotizante y/o la muerte del paciente ${ }^{11}$. El tratamiento debe regirse en cuatro principios, los cuales deben realizarse inmediatamente: manejo de la vía aérea, drenaje extraoral y/o intraoral más exodoncia o tratamiento conservador de la pieza causante (apertura cameral), terapia antibiótica temprana agresiva y soporte adecuado de nutrición e hidratación ${ }^{11}$.
Se presenta un caso de angina de Ludwig, tratado con éxito en el servicio de cirugía bucal y maxilofacial del Hospital Regional Honorio Delgado de Arequipa, Perú.

\section{Reporte del caso}

Paciente mujer de 32 años, quien manifestó tener antecedentes de anemia, relacionada a una alteración de duración del ciclo menstrual, diagnóstico dado en otro centro hospitalario. Refirió odontalgia en pieza dentaria en maxilar inferior por lo que fue a consultorio particular donde le prescribieron analgésicos sin presentar mejorías. Cuatro días antes del ingreso notó un aumento de volumen facial, malestar general, disfagia, sensación de alza térmica. Acudió por emergencia del Hospital Regional Honorio Delgado por lo que fue evaluada por el servicio de cirugía bucal y maxilofacial.

La evaluación inicial reportó una temperatura de $37,8^{\circ} \mathrm{C}$, frecuencia cardiaca de 110 lat $/ \mathrm{min}$, frecuencia respiratoria de 20 por minuto, saturación de oxígeno al $99 \%$. La auscultación pulmonar descartó signos de compromiso pulmonar. Apoyados en criterios clínicos, como la ausencia de disnea, estridor laríngeo y los signos vitales estables respecto al sistema respiratorio, se descartó el compromiso de la vía aérea. El examen clínico extraoral evidenció una asimetría facial por un aumento de volumen a nivel de la región submandibular bilateral y región submental. (Figuras 1A, B y C). A la palpación, el aumento de volumen tenía una consistencia blanda, fluctuante, con signos flogóticos, rubor, calor y dolor. A la evaluación intraoral, la apertura bucal estaba limitada $(4 \mathrm{~mm})$, mala higiene bucodental, protrusión lingual, aumento de volumen de piso de boca que comprometía la región sublingual bilateral y pieza dentaria 3.7 con destrucción coronaria (Figuras 1D y E), por lo que se indicó su hospitalización. La evaluación clínica y la historia del paciente llevaron al diagnóstico de angina de Ludwig, siendo la pieza 3.7 la desencadenante del proceso.

Se solicitaron exámenes de laboratorio, tomografía computarizada cervical - cuello y radiografía panorámica. Los exámenes indicaron valores de hemoglobina de 7,2 $\mathrm{g} / \mathrm{dl}$, leucocitos de $18200 \mathrm{u} / \mathrm{mm}^{3}$, segmentados $85 \%$, plaquetas $350000 \mathrm{~mm}^{3}$, tiempo de protrombina 16 " 4 , tiempo parcial de tromboplastina 34 "9 y un INR (índice internacional normalizado) de 1,45. La radiografía panorámica evidenció una imagen radiolúcida a nivel apical y destrucción coronaria a nivel disto oclusal con compromiso pulpar de pieza 3.7 (Figura 2A). La evaluación tomográfica mostró un engrosamiento de las paredes de la orofaringe piso de boca y colección purulenta a nivel de región submandibular y submental. Se confirmó desviación de vía aérea, con predominio de esta hacia el lado derecho. Disminución de vía aérea, pero sin alteración de la permeabilidad (Figuras 2B, C y D).

Inmediatamente, bajo anestesia local, se realizó drenaje intraoral a través de incisiones lineales en fondo de surco vestibular y piso de boca. Drenaje extraoral con incisiones lineales siguiendo las líneas de tensión facial de Langer, a nivel de región submandibular bilateral y submental. Se colocaron drenes Penrose a nivel de regiones 


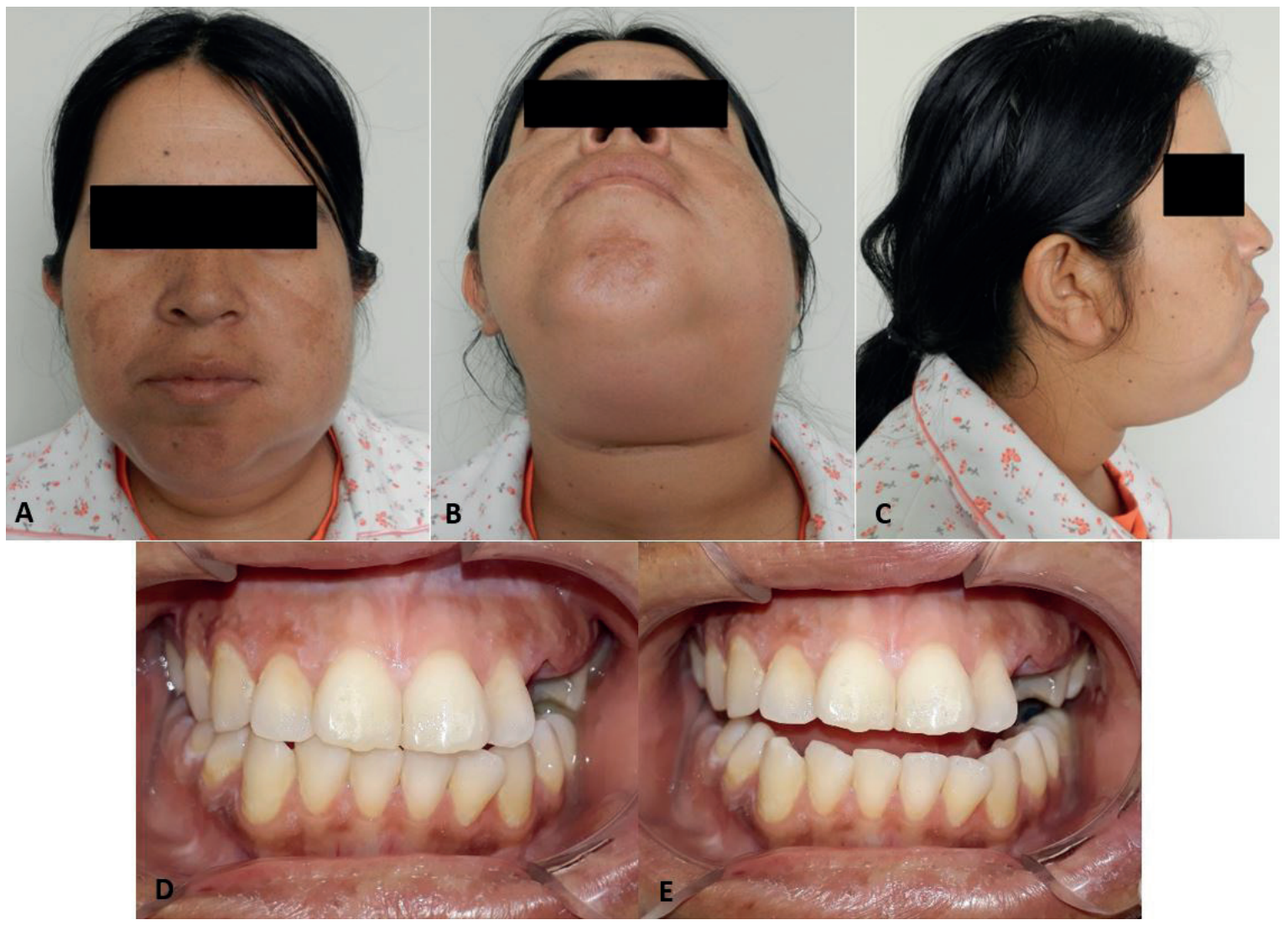

Figura 1. A, B, y C: asimetría facial por aumento de volumen en región submandibular bilateral y submental. D y E: mala higiene oral, aumento de volumen a nivel de piso de boca que compromete región sublingual, apertura bucal limitada (aproximadamente $4 \mathrm{~mm}$ )
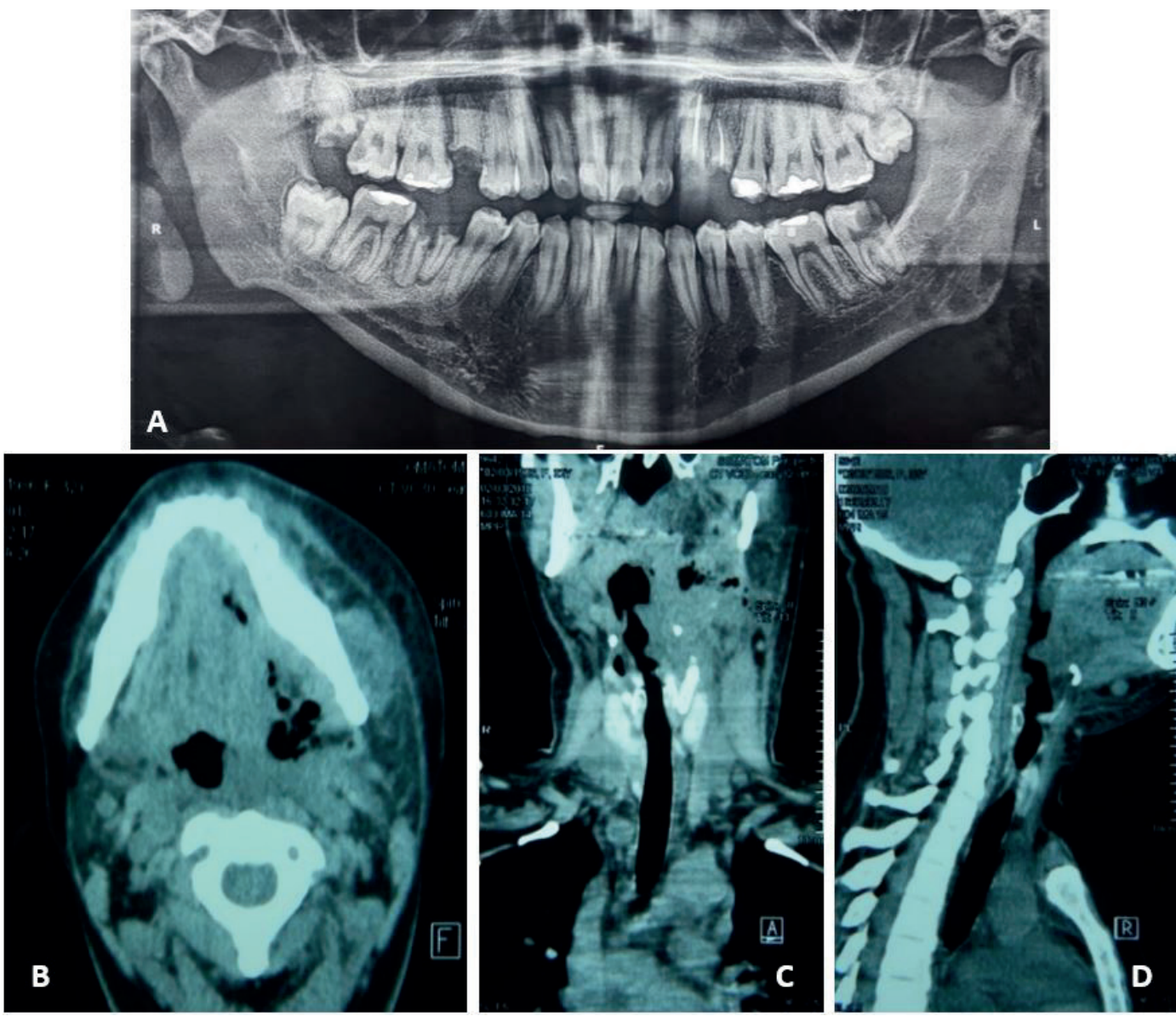

Figura 2. A: radiografía panorámica muestra imágenes radiolúcidas a nivel apical de pieza 3.7 correspondiente a proceso infeccioso. B, C y D: cortes tomográficos que evidencian imágenes hipodensas compatibles a engrosamiento de paredes y colección a nivel de espacios submandibulares, sublinguales y submental. Vía aérea permeable 
donde se realizó el drenaje extraoral y se extrajeron las piezas 3.7 y 4.6 (Figuras 3A y B). Se tomó una muestra de secreción purulenta para cultivo y antibiograma. Se instauró antibioticoterapia con clindamicina $600 \mathrm{mg}$ c/8 h vía endovenosa y ceftriaxona $1 \mathrm{~g} \mathrm{c} / 12 \mathrm{~h}$ vía endovenosa. Se realizaron interconsultas al servicio de infectología, medicina interna y ginecoobstetricia.

Infectología y medicina interna descartaron compromiso sistémico relacionado a la anemia y sugirieron transfusión sanguínea. Ginecoobstetricia atribuyó anemia a una alteración en el ciclo menstrual, el cual terminó dos días antes de ingreso de la paciente. También sugirió transfusión de paquetes globulares y control ambulatorio posterior a manejo de proceso infeccioso. Se transfundieron dos paquetes globulares, inmediatamente después del procedimiento quirúrgico. Se realizaron curaciones diarias, cambio de drenes y apósitos compresivos.

Los resultados del cultivo aislaron solo Stresptococcus mutans y el antibiograma fue sensible a clindamicina y ceftriaxona. Cinco días posteriores al tratamiento quirúrgico y antibioticoterapia el paciente se encontró en mejores condiciones. Los nuevos exámenes indicaron un hemograma dentro de los parámetros normales. Hemoglobina de $12,5 \mathrm{~g} / \mathrm{dl}$, leucocitos de $6300 \mathrm{u} / \mathrm{mm}^{3}$, segmentados de $65 \%$. Ausencia de sintomatología y de secreción purulenta. La paciente evolucionó favorablemente y fue dada de alta. Se prescribió clindamicina $300 \mathrm{mg}$ c/8 h vía oral por cuatro días. Acudió a sus controles dos días, siete y quince días posterior al alta. No se presentó ninguna complicación asociada al tratamiento (Figura 4).

\section{Discusión}

Debido a que el manejo de la vía aérea es el factor más importante en pacientes con angina de Ludwig, descartar su obstrucción será el primer objetivo ${ }^{10}$. El mantenimiento de esta a través de la traqueostomía o técnicas avanzadas de vía aérea, como posicionamiento vertical, intubación despierta, intubación con fibra óptica, e in- tubación retrógrada, frente a la observación, van a depender del estado actual del paciente ${ }^{12,13}$. El cual será determinado por el juicio clínico del tratante ${ }^{12}$. En el caso presentado la vía aérea de la paciente estaba permeable, se corroboró a través de los signos vitales y la evaluación clínica. Por lo cual, se inició inmediatamente el tratamiento quirúrgico y antibioticoterapia, evitando la morbilidad de realizar una traqueostomía.

No se administró corticoides, debido a la ausencia de justificación para mejorar la vía aérea, ya que estaba permeable. Su uso está contraindicado en pacientes con anemia ${ }^{1,11,14}$. Aún es controversial su protocolización como adyuvante en el tratamiento de infecciones odontogénicas. Fong realizó un estudio retrospectivo donde concluyó que no existe evidencia suficiente para usar corticoides en pacientes con infecciones odontogénicas y sugirió evitar su uso en pacientes inmunosuprimidos o con enfermedades sistémicas ${ }^{14}$. Coincidimos, ya que solo su uso se justificaría en mejorar la vía aérea cuando se requiera intubación urgente. En el caso presentado la vía aérea estaba permeable por lo cual no era justificado. No es un protocolo estándar en nuestra práctica diaria.

El gold standard en el tratamiento de la angina de Ludwig una vez mantenida la vía aérea es el drenaje extraoral e intraoral y la exodoncia o tratamiento inmediato de la pieza dentaria causante ${ }^{15}$. Igoumenakis realizó un estudio y demostró la importancia de realizar la exodoncia de la pieza involucrada en infecciones odontogénicas graves, disminuyendo significativamente los días de hospitalización, el número de leucocitos y mejorando el estado sistémico ${ }^{15}$.

Los antibióticos cumplen un papel importante pero no protagónico. Son excelentes adyuvantes. Reducen el tiempo de resolución significativamente y se debe saber cuándo prescribirlos sino, no se dará ninguna posibilidad al organismo de controlar la infección, contribuir a la selección de bacterias resistentes y someter al paciente a los muchos efectos adversos ${ }^{16}$. Se estará contribuyen-
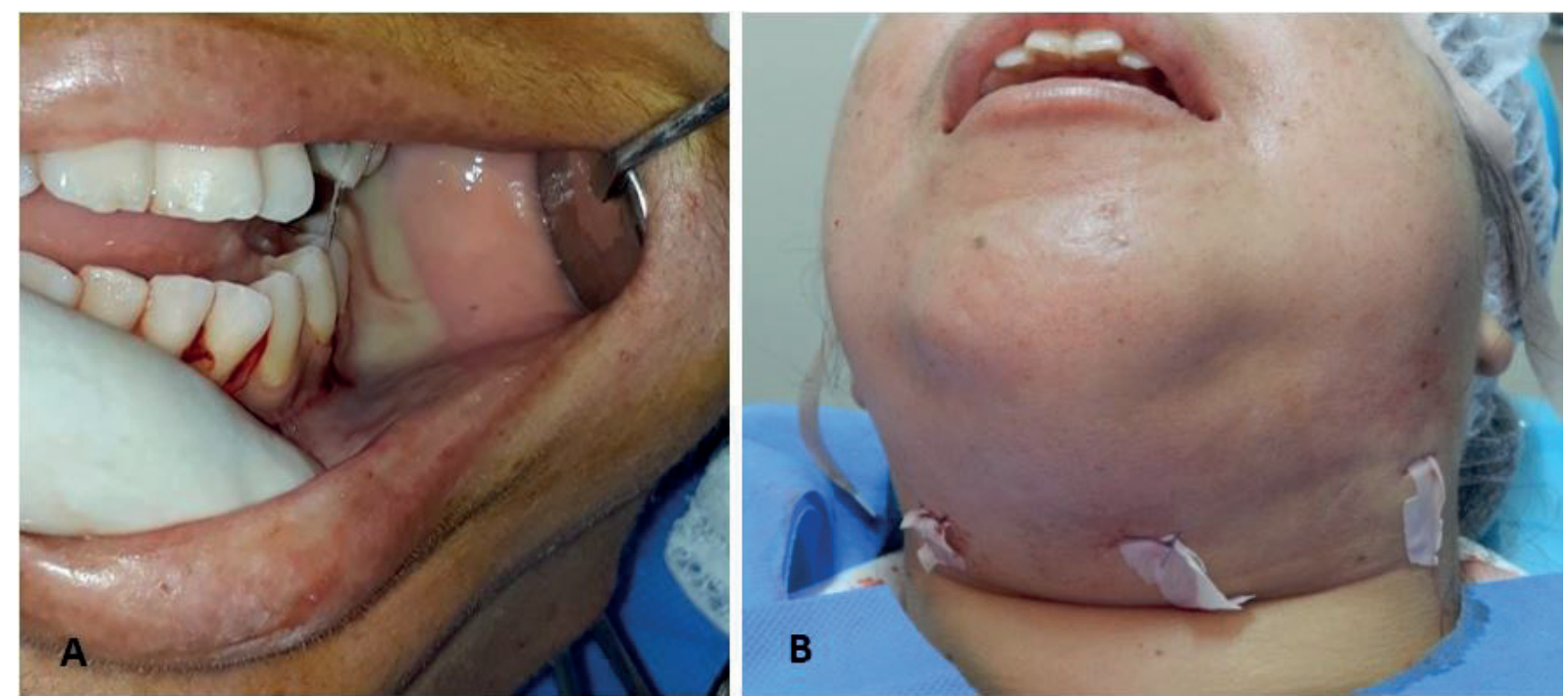

Figura 3. A: drenaje intraoral, se evidencia secreción purulenta. B: drenaje extraoral a nivel de región submandibular bilateral y submental, colocación de drenes Penrose 


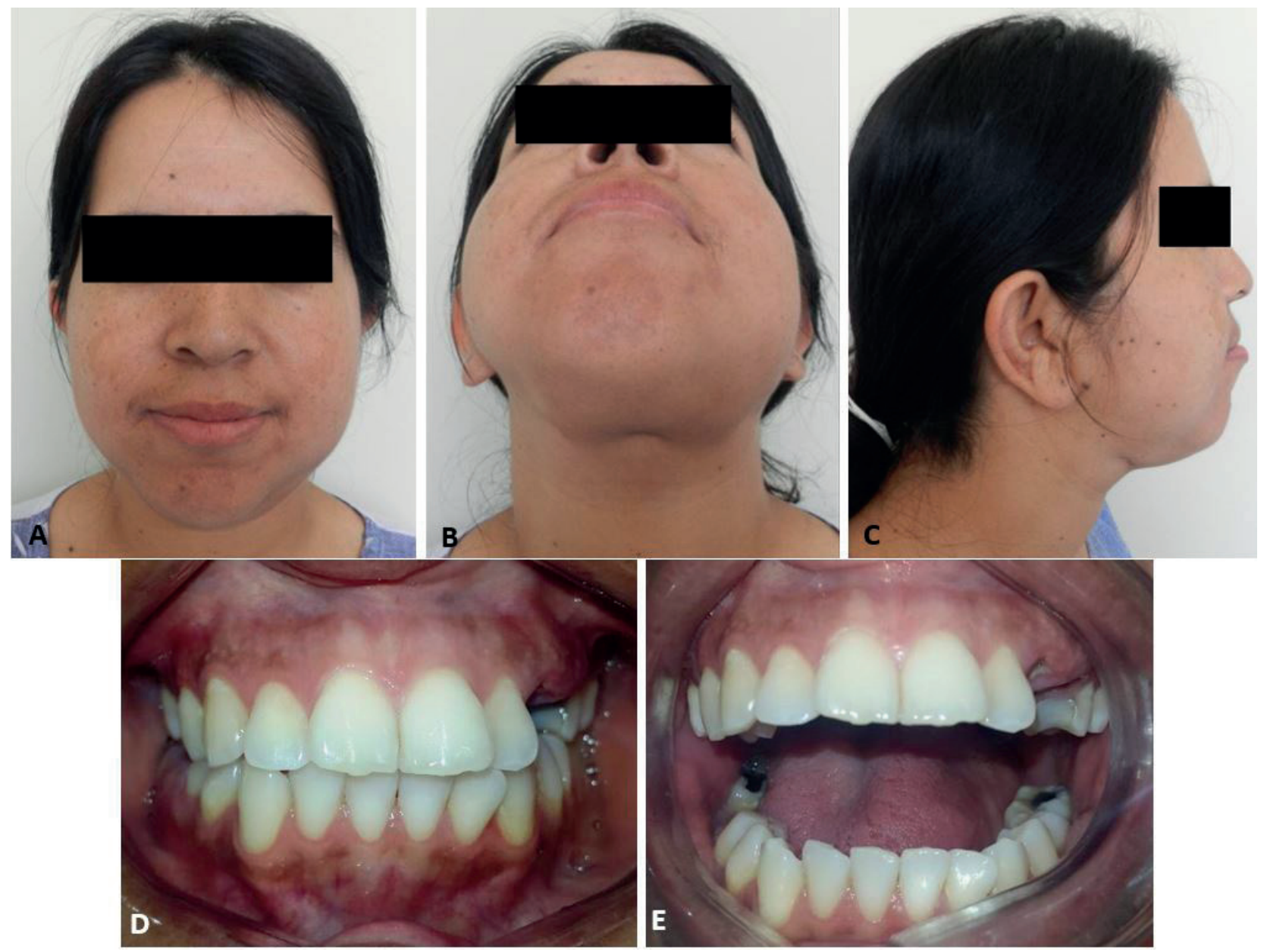

Figura 4. A, B y C: vista extraoral de control posterior a tratamiento. D y E: evaluación intraoral que muestra la ausencia de aumento de volumen de piso de boca, buena higiene oral y apertura bucal conservada

do indebidamente al creciente problema de la resistencia bacteriana. Es importante considerar su individualización. Cuando el drenaje y/o la eliminación de la causa de la infección se llevan a cabo correctamente, todos los antibióticos son igualmente efectivos con respecto a la cura clínica ${ }^{15}$. Flynn y cols. ${ }^{1}$ recomiendan la instauración empírica de antibióticos, con el uso de las penicilinas, clindamicina, cefalosporinas de segunda generación como la ceftriaxona o la combinación de penicilinas con ácido clavulánico o sulbactam, recalcando que mientras no se trate el factor etiológico no se obtendrá los resultados esperados ${ }^{1,11}$. Nosotros instauramos una antibioticoterapia empírica con clindamicina y ceftriaxona, hasta esperar el resultado del cultivo y antibiograma, el cual coincidió con la prescripción instaurada; teniendo resultados óptimos. La terapia antibiótica continuará hasta que se informen los resultados de los cultivos y las pruebas de sensibilidad, en cuyo momento se pueden hacer ajustes apropiados en los regímenes terapéuticos ${ }^{17}$.

La angina de Ludwig es una infección potencialmente mortal. Un retraso en el diagnóstico o un inadecuado manejo, puede desencadenar complicaciones sistémicas, secuelas irreversibles, potencialmente fatales. Es de vital importancia conocer las manifestaciones clínicas y sisté- micas de esta patología ya que nos llevan a realizar un diagnóstico temprano y por ende un tratamiento exitoso.

\section{Referencias bibliográficas}

1. Flynn T, Shanti R, Levi M, Adamo A. Severe odontogenic infections, part 1: prospective report. J Oral Maxillofac Surg. 2006;64(6):1093-103.

2. Wasson J, Hopkins C, Bowdler D. Did Ludwig's angina kill Ludwig? J Laryngol Otol. 2006;120:363-365.

3. Nguyen V, Potter J, Hersh-Schick M. Ludwig angina: an uncommon and potentially lethal neck infection. Am J Neuroradiol.1992;13:215-9.

4. Saifeldeen K, Evans R. Ludwig's angina. Emerg Med J. 2004;21(3):242-3.

5. Bansal A, Miskoff J, Lis R. Otolaryngologic critical care. Crit Care Clin. 2003;19:55-72.

6. Daramola O, Flanagan C, Maisel R. Diagnosis and treatment of deep neck space abscesses. Otolaryngol Head Neck Surg. 2009;141:123-30.

7. Boscolo-Rizzo P, Da Mosto M. Submandibular space infection: a potentially lethal infection. Int J Infect Dis. 2009;3:327-33. 
8. Chou Y, Lee Y, Chao H. An upper airway obstruction emergency: Ludwig's angina. Pediatr Emerg Care. 2007;23:892-6.

9. Kremer M, Blair T. Ludwig angina: forewarned is forearmed. J Am Assoc Nurse Anesth. 2006;74(4):445-51.

10. Wolfe M, Davis J, Parks S. Is surgical airway necessary for airway management in deep neck infections and Ludwig angina? J Crit Care. 2011;26:11-4.

11. Flynn TR, Shanti RM, Hayes C. Severe odontogenic infections, part two: prospective outcomes study. J Oral Maxillofac Surg. 2006;64(6):1104-13.

12. Potter J, Herford A, Ellis E. Tracheotomy versus endotracheal intubation for airway management in deep neck space infections. J Oral Maxillofac Surg. 2002;60(4):349-54.

13. Hagberg C. Special devices and techniques. Anesthiology Clin N Am. 2002;20:907-32.
14. Fong L, Harshinie A, Hui Woon L. Steroids in Maxillofacial Space Infection: A Retrospective Cohort Study. Open J Stomatol. 2017;7:397-407.

15. Igoumenakis D, Giannakopoulos N. Effect of Causative Tooth Extraction on Clinical and Biological Parameters of Odontogenic Infection: A Prospective Clinical Trial. J Oral Maxillofac Surg. 2015;73:1254-8.

16. Martins J, Chagas O. The Use of Antibiotics in Odontogenic Infections: What Is the Best Choice? A Systematic Review. J Oral Maxillofac Surg. 2017;75:2606. e1-2606.e11.

17. Farmahan S, Tuopar D, Ameerally J. The clinical relevance of microbiology specimens in head and neck space infections of odontogenic origin. Br J Oral Maxillofac Surg. 2014;52:629. 